\title{
Unobserved component models with asymmetric conditional variances
}

\author{
Carmen Broto ${ }^{\mathrm{a}, \mathrm{b}, *}$, Esther Ruiz ${ }^{\mathrm{a}}$ \\ ${ }^{a}$ Departamento de Estadística, Universidad Carlos III de Madrid, C/Madrid 126, 28903 Getafe, Spain \\ ${ }^{\mathrm{b}}$ Servicio de Estudios BBVA, Castellana 81, 28046 Madrid, Spain
}

\begin{abstract}
Unobserved component models with GARCH disturbances are extended to allow for asymmetric responses of conditional variances to positive and negative shocks. The asymmetric conditional variance is represented by a member of the QARCH class of models. The proposed model allows to distinguish whether the possibly asymmetric conditional heteroscedasticity affects the short-run or the long-run disturbances or both. Statistical properties of the new model and the finite sample properties of a QML estimator of the parameters are analyzed. The correlogram of squared auxiliary residuals is shown to be useful to identify the conditional heteroscedasticity. Finite sample properties of squared auxiliary residuals are also analysed. Finally, the results are illustrated by fitting the model to daily series of financial and gold prices, as well as to monthly series of inflation. The behavior of volatility in both types of series is different. The conditional heteroscedasticity mainly affects the short-run component in financial prices while in the inflation series, the heteroscedasticity appears in the long-run component. Asymmetric effects are found in both types of variables.
\end{abstract}

Keywords: Auxiliary residuals; Financial series; GARCH; Inflation; Leverage effect; QARCH; Structural time series models

\footnotetext{
* Corresponding author. Servicio de Estudios BBVA, Castellana 81, Madrid 28046, Spain. Tel.: 34915373977 ; fax: 34916249849.

E-mail address: carmen.broto@grupobbva.com (C. Broto).
} 


\section{Introduction}

Economic time series can often be decomposed into components that have a direct interpretation, for example, trend, seasonal and transitory components; see Harvey (1989) for a detailed description of unobserved component models. In the simplest case, the series of interest, $y_{t}$, can be decomposed in a long-run component, representing an evolving level, $\mu_{t}$, and a transitory component, $\varepsilon_{t}$. If the level follows a random walk and the transitory component is white noise, the resulting model is given by

$$
\begin{aligned}
& y_{t}=\mu_{t}+\varepsilon_{t}, \\
& \mu_{t}=\mu_{t-1}+\eta_{t},
\end{aligned}
$$

where $\varepsilon_{t}$ and $\eta_{t}$ are mutually independent Gaussian white noise processes with variances $h$ and $q$ respectively. Model (1) is known as random walk plus noise and has been very useful to represent the dynamic dependence of a large number of economic time series; see, for example, Durbin and Koopman (2001) for several applications concerning this model.

The random walk plus noise model in (1) was extended by Harvey et al. (1992) to allow the variances of both, the short- and the long-run components, to evolve over time following $\operatorname{GARCH}(1,1)$ models. In particular, the disturbances are defined by $\varepsilon_{t}=\varepsilon_{t}^{\dagger} h_{t}^{1 / 2}$ and $\eta_{t}=\eta_{t}^{\dagger} q_{t}^{1 / 2}$ where $\varepsilon_{t}^{\dagger}$ and $\eta_{t}^{\dagger}$ are mutually independent Gaussian white noise processes and $h_{t}$ and $q_{t}$ are given by

$$
\begin{aligned}
& h_{t}=\alpha_{0}+\alpha_{1} \varepsilon_{t-1}^{2}+\alpha_{2} h_{t-1}, \\
& q_{t}=\gamma_{0}+\gamma_{1} \eta_{t-1}^{2}+\gamma_{2} q_{t-1},
\end{aligned}
$$

where the parameters $\alpha_{0}, \alpha_{1}, \alpha_{2}, \gamma_{0}, \gamma_{1}$ and $\gamma_{2}$ satisfy the usual conditions to guarantee the positivity and stationarity of $h_{t}$ and $q_{t}$.

Model (1) with the variances defined as in (2) is a Structural ARCH (STARCH) model. The main attractive of STARCH models is that they are able to distinguish whether the ARCH effects appear in the permanent and/or in the transitory component. An alternative heteroscedastic unobserved component model is proposed by Ord et al. (1997), where, instead of considering different disturbances for each component, the source of randomness is unique.

Unobserved component models with GARCH disturbances have been applied in fields like macroeconomics and finance. For example, Evans and Wachtel (1993), Ball and Cecchetti (1990) and Evans (1991) analyze inflation, Kim (1993) analyzes inflation and interest rates, Fiorentini and Maravall (1996) analyze the Spanish money supply and Diebold and Nerlove (1989), King et al. (1994), Morgan and Trevor (1999), Hasbrouck (1999), Bos et al. (2000) and Wei (2002) have applications to financial data.

The variances in Eqs. (2) are specified in such a way that their responses to positive and negative changes in the corresponding disturbances are symmetric. However, in some cases, the empirical evidence suggest that the conditional variance may have a different response to shocks of the same magnitude but different sign. This phenomenon, known as "leverage effect" in the Financial Econometrics literature, has often been observed in high frequency financial data; see, for example, Shephard (1996) and the references therein. In the 
context of macroeconomic time series, Brunner and Hess (1993), point out the importance of considering the "leverage effect" in the modelization of inflation.

There are several alternative models proposed in the literature to represent asymmetric responses of volatility to positive and negative shocks; see Hentschel (1995) and He and Teräsvirta (1999) for two asymmetric models that encompass many of the most popular alternatives. In this paper, we consider the Generalized Quadratic ARCH (GQARCH) model originally proposed by Sentana (1995) because of its tractability in the sense that GQARCH models pick up the "leverage effect" in an additive way. Consequently, the estimation of these models is easier than in models that use a multiplicative specification like, for example, the EGARCH model of Nelson (1991). If the disturbances $\varepsilon_{t}$ and $\eta_{t}$ follow GQARCH(1,1) processes, their variances are given by

$$
\begin{aligned}
& h_{t}=\alpha_{0}+\alpha_{1} \varepsilon_{t-1}^{2}+\beta \varepsilon_{t-1}+\alpha_{2} h_{t-1}, \\
& q_{t}=\gamma_{0}+\gamma_{1} \eta_{t-1}^{2}+\delta \eta_{t-1}+\gamma_{2} q_{t-1},
\end{aligned}
$$

respectively. The parameters in (3) should be restricted for the variances to be positive. In particular $\alpha_{0}, \alpha_{1}, \alpha_{2}>0$ and $\beta^{2} \leqslant 4 \alpha_{1} \alpha_{0}$. On the other hand, $\varepsilon_{t}$ is covariance stationary if $\alpha_{1}+\alpha_{2}<1$. Similar restrictions are imposed on $\gamma_{0}, \gamma_{1}, \delta$ and $\gamma_{2}$; see He and Teräsvirta (1999).

Sentana (1995) analyzes the properties of the GQARCH(1,1) model and points out their similarity to the $\operatorname{GARCH}(1,1)$ model. For example, the $\operatorname{GARCH}(1,1)$ and $\operatorname{GQARCH}(1,1)$ models for $\varepsilon_{t}$ have the same unconditional mean and variance equal to zero and $\sigma_{\varepsilon}^{2}=$ $\alpha_{0} /\left(1-\alpha_{1}-\alpha_{2}\right)$, respectively. Furthermore, in both models, the odd moments are zero, the series $\varepsilon_{t}$ is uncorrelated and the cross-correlations between $\varepsilon_{t}^{2}$ and $\varepsilon_{t-h}$ are zero for all $h \geqslant 2$. When $h=1, \operatorname{Cov}\left(\varepsilon_{t}^{2}, \varepsilon_{t-1}\right)=\beta \sigma_{\varepsilon}^{2}$ in the $\operatorname{GQARCH}(1,1)$ model and zero in the $\operatorname{GARCH}(1,1)$ model. Using the results of He and Teräsvirta (1999), it is possible to derive the following expressions for the kurtosis of $\varepsilon_{t}$ and autocorrelation function (acf) of $\varepsilon_{t}^{2}$

$$
\kappa_{\varepsilon}=\frac{3\left(1-\left(\alpha_{1}+\alpha_{2}\right)^{2}\right)}{\left(1-3 \alpha_{1}^{2}-\alpha_{2}^{2}-2 \alpha_{1} \alpha_{2}\right)}+3 \frac{A^{*}}{\left(1-3 \alpha_{1}^{2}-\alpha_{2}^{2}-2 \alpha_{1} \alpha_{2}\right)}
$$

and

$$
\rho_{\varepsilon^{2}}(\tau)= \begin{cases}\frac{2 \alpha_{1}\left(1-\alpha_{1} \alpha_{2}-\alpha_{2}^{2}\right)+A^{*}\left(3 \alpha_{1}+\alpha_{2}\right)}{2\left(1-2 \alpha_{1} \alpha_{2}-\alpha_{2}^{2}\right)+3 A^{*}}, & \tau=1, \\ \left(\alpha_{1}+\alpha_{2}\right)^{\tau-1} \rho_{\varepsilon^{2}}(1), & \tau>1,\end{cases}
$$

where $A^{*}=\left(\beta / \sigma_{\varepsilon}\right)^{2}$. Notice that the kurtosis of $\varepsilon_{t}$ is larger than in the symmetric GARCH model. For example, if $\alpha_{0}=0.05, \alpha_{1}=0.15, \alpha_{2}=0.8$ and $\beta=0$, the kurtosis is 5.57 while if, for the same parameter values, $|\beta|=0.1$, the kurtosis is 6.14. On the other hand, the autocorrelation function of the squares of a $\operatorname{GQARCH}(1,1)$ model decays at the same rate as in the $\operatorname{GARCH}(1,1)$ model. Furthermore, if $\beta$ is small relative to $\sigma_{\varepsilon}^{2}$, as it is usually the case in empirical applications, the autocorrelation of order one is almost the same in both models. For example, for the same parameter values considered before, if $\beta=0$, then $\rho_{\varepsilon^{2}}(1)=0.3$ while if $|\beta|=0.1$, then $\rho_{\varepsilon^{2}}(1)=0.31$. Therefore, it seems that incorporating the leverage effect into the conditional variance increases the kurtosis of the process without increasing the autocorrelations of squares. 
The objective of this paper is to extend the STARCH model by allowing the variances of the disturbances $\varepsilon_{t}$ and $\eta_{t}$ to follow GQARCH models. Hereafter, we call this new family of models Quadratic STARCH (Q-STARCH). These models are able to represent asymmetric responses of conditional variances to positive and negative disturbances distinguishing whether the asymmetry appears in the short- or in the long-run components. Secondly, we will show how the autocorrelations of the squared auxiliary residuals can be used to identify which of these components is conditionally heteroscedastic.

It is important to mention that alternatively, the evolution of the variances of $\varepsilon_{t}$ and $\eta_{t}$ could be modelled using stochastic volatility (SV) models instead of the GARCH type of models chosen in this paper. However, in this case it is necessary to restrict the dynamic of the variances to estimate the models. For example, Koopman and Bos (2004) propose a model with unobserved SV components but they restrict all the components to have the same dynamics for the variances. On top of this, the estimation of the parameters of the models with SV components requires more complicated methods than when the disturbances belong to the family of GARCH models.

The paper is organized as follows. Section 2 introduces the Q-STARCH model and describes its properties. It also contains finite sample properties of the autocorrelations of squared observations and squared auxiliary residuals, which are useful to identify the presence of heteroscedastic asymmetric variances. In Section 3, we analyze the finite sample properties of a quasi-maximum likelihood (QML) estimator of the parameters of the QSTARCH model based on the prediction error decomposition of the Gaussian log-likelihood. In Section 4, the Q-STARCH model is fitted to daily gold and financial prices and to monthly series of inflation. Finally, Section 5 concludes the paper.

\section{Q-STARCH model}

In this section, we analyze the statistical properties of the Q-STARCH model defined by Eqs. (1) and (3). The stationary form of the model is given by

$$
\Delta y_{t}=\eta_{t}+\Delta \varepsilon_{t} .
$$

From (6) it can be easily seen that $y_{t}$ follows an ARIMA $(0,1,1)$ with non-Gaussian innovations. Furthermore, notice that the innovations of this model are uncorrelated although not independent; see Breidt and Davis (1992). The mean, variance and acf of $\Delta y_{t}$ are the same as in the homoscedastic random walk plus noise model; see, for example, Harvey (1989). The presence of asymmetric ARCH effects is reflected in the kurtosis of $\Delta y_{t}$ given by

$$
\begin{aligned}
\kappa\left(\Delta y_{t}\right)= & \frac{3}{(q+2)^{2}}\left\{4 q+q^{2} \frac{1-\left(\gamma_{1}+\gamma_{2}\right)^{2}+B^{*}}{1-3 \gamma_{1}^{2}-2 \gamma_{1} \gamma_{2}-\gamma_{2}^{2}}\right. \\
& \left.+\frac{4\left(1-\left(\alpha_{1}+\alpha_{2}\right)^{2}+\alpha_{1}\left(1-\alpha_{1}-\alpha_{1} \alpha_{2}-\alpha_{2}^{2}\right)\right)+2 A^{*}\left(1+3 \alpha_{1}+\alpha_{2}\right)}{1-3 \alpha_{1}^{2}-2 \alpha_{1} \alpha_{2}-\alpha_{2}^{2}}\right\},
\end{aligned}
$$


where $q=\sigma_{\eta}^{2} / \sigma_{\varepsilon}^{2}, \sigma_{\eta}^{2}=\gamma_{0} /\left(1-\gamma_{1}-\gamma_{2}\right)$ and $B^{*}=\left(\delta / \sigma_{\eta}\right)^{2}$. Notice that, in the homoscedastic case, when $\alpha_{1}=\gamma_{1}=\beta=\delta=0$, the kurtosis is, as expected, 3. The presence of ARCH effects, $\alpha_{1} \neq 0$ or $\gamma_{1} \neq 0$, causes excess kurtosis. Besides, in the asymmetric case, when $\beta \neq 0$ or $\delta \neq 0$, the excess kurtosis is even greater. Therefore, the kurtosis of a Q-STARCH model is bounded from bellow by the kurtosis of a symmetric STARCH model independently of the sign of $\beta$ or $\delta$. The kurtosis is, in general, a complicated function of the signal to noise ratio, $q$, and of the ARCH parameters. For example,assuming that the ARCH effects of the long and short-run disturbances are identical, i.e. $\gamma_{0}=\alpha_{0}, \gamma_{1}=\alpha_{1}$ and $\gamma_{2}=\alpha_{2}$, the kurtosis increases more when the asymmetry of the transitory component increases $(\beta)$ than when the asymmetry of the long-run disturbance increases $(\delta)$.

Furthermore, the skewness of $\Delta y_{t}$ is given by

$$
\operatorname{SK}\left(\Delta y_{t}\right)=\frac{-3 \beta}{\sigma_{\varepsilon}(q+2)^{3 / 2}}
$$

Note that only the asymmetry of the transitory component, $\beta$, affects the skewness coefficient. Looking at expressions (7) and (8), it seems that, independently of the signal to noise ratio, $q$, the asymmetries of the transitory noise are more influential than the asymmetries of the long-run noise on the statistical properties of $\Delta y_{t}$. In any case, the main dynamic properties of $\left(\Delta y_{t}\right)$ appear in the squares. After some tedious algebra, it is possible to derive the following expression of the autocovariance function of $\left(\Delta y_{t}\right)^{2}$

$$
\gamma_{\left(\Delta y_{t}\right)^{2}}(\tau)= \begin{cases}\sigma_{\varepsilon}^{4}(q+2)^{2}\left(\kappa\left(\Delta y_{t}\right)-1\right), & \tau=0, \\ \sigma_{\varepsilon}^{4}\left\{q^{2}\left(\kappa_{\eta}-1\right) \rho_{\eta^{2}}(1)\right. & \\ \left.+\left(\kappa_{\varepsilon}-1\right)\left[1+\left(2+\alpha_{1}+\alpha_{2}\right) \rho_{\varepsilon^{2}}(1)\right]\right\}, & \tau=1, \\ \left(\alpha_{1}+\alpha_{2}\right) \gamma_{\left(\Delta y_{t}\right)^{2}(\tau-1)} & \\ +\left[\left(\gamma_{1}+\gamma_{2}\right)-\left(\alpha_{1}+\alpha_{2}\right)\right]\left(\gamma_{1}+\gamma_{2}\right)^{\tau-2} \gamma_{\eta^{2}}(1), & \tau \geqslant 2,\end{cases}
$$

where $\kappa\left(\Delta y_{t}\right)$ is given in (7) and $\rho_{\eta^{2}}(1)$ and $\gamma_{\eta^{2}}(1)$ are the autocorrelation and autocovariance of order one of $\eta_{t}^{2}$, respectively. From (9), it is straightforward to obtain the acf of $\left(\Delta y_{t}\right)^{2}$. Notice that the decay in the correlogram of the squared first differences is the same as for the symmetric STARCH model. Furthermore, when the persistence of the variances of the short- and long-run components is similar, the decay of the autocorrelations is exponential with parameter $\alpha_{1}+\alpha_{2}$. As expected, in the homoscedastic case, when $\alpha_{1}=\gamma_{1}=\beta=\delta=0$, all the autocorrelations for $\left(\Delta y_{t}\right)^{2}$ are zero for lags greater than one and the autocorrelation at lag one is $(1 /(q+2))^{2}=\left[\rho_{\Delta y_{t}}(1)\right]^{2}$, where $\rho_{\Delta y_{t}}(1)$ is the lag one autocorrelation of $\Delta y_{t}$. Therefore, the autocorrelations of the squared observations are equal to the squared autocorrelations of the row observations; see Maravall (1983).

The information about the asymmetric response of the variances to positive and negative innovations is more evident in the cross-correlations between $\left(\Delta y_{t}\right)^{2}$ and $\Delta y_{t-\tau}$ that are 
given by

$$
\operatorname{Corr}_{\left[\left(\Delta y_{t}\right)^{2}, \Delta y_{t-\tau}\right]}= \begin{cases}0, & \forall \tau<-1, \\ \frac{2 \beta}{\sigma_{\varepsilon}(q+2)^{3 / 2}\left(\kappa\left(\Delta y_{t}\right)-1\right)^{1 / 2}}, & \tau=-1, \\ \frac{-3 \beta}{\sigma_{\varepsilon}(q+2)^{3 / 2}\left(\kappa\left(\Delta y_{t}\right)-1\right)^{1 / 2}}, & \tau=0, \\ \frac{\delta q-\beta\left(\alpha_{1}+\alpha_{2}\right)}{\sigma_{\varepsilon}(q+2)^{3 / 2}\left(\kappa\left(\Delta y_{t}\right)-1\right)^{1 / 2}}, & \tau=1, \\ \frac{\delta\left(\gamma_{1}+\gamma_{2}\right)^{\tau-1} q}{\sigma_{\varepsilon}(q+2)^{3 / 2}\left(\kappa\left(\Delta y_{t}\right)-1\right)^{1 / 2}}+ & \\ \frac{\beta\left(\left(\alpha_{1}+\alpha_{2}\right)^{\tau-2}-\left(\alpha_{1}+\alpha_{2}\right)^{\tau}\right)}{\sigma_{\varepsilon}(q+2)^{3 / 2}\left(\kappa\left(\Delta y_{t}\right)-1\right)^{1 / 2}}, & \tau \geqslant 2 .\end{cases}
$$

Note that in the symmetric STARCH case these third order moments are always zero. In any case, given that the asymmetry parameters, $\beta$ and $\delta$, are rather small in magnitude, the size of the cross-correlations is so small that they are not an useful instrument to identify the presence of asymmetries.

In unobserved component models, it can also be useful to analyze the auxiliary residuals, that estimate the disturbances of each component; see Maravall (1987) and Harvey and Koopman (1992). The latter authors show that the minimum mean square linear (MMSL) estimators of $\varepsilon_{t}$ and $\eta_{t}$ are given by

$$
\begin{aligned}
& \widehat{\eta}_{t}=\frac{(1+\theta)^{2} \Delta y_{t}}{(1-\theta L)(1+\theta F)}, \\
& \widehat{\varepsilon}_{t}=\frac{\theta}{1+\theta^{2}}\left(\widehat{\eta}_{t+1}-\widehat{\eta}_{t}\right),
\end{aligned}
$$

where $F$ is the lead operator such that $F x_{t}=x_{t+1}, L$ is the lag operator such that $L x_{t}=$ $x_{t-1}$ and $\theta$ is the moving average parameter of the reduced form of $\Delta y_{t}$ given by $\theta=$ $\left(-q-2+\sqrt{q^{2}+4 q}\right) / 2$. Harvey and Koopman (1992) show that, if time is reversed, $\widehat{\eta}_{t}$ follows an AR(1) model with parameter $\theta$ whereas $\widehat{\varepsilon}_{t}$ follows a strictly noninvertible $\operatorname{ARMA}(1,1)$ process with autoregressive parameter $\theta$. The first order autocorrelation of $\widehat{\varepsilon}_{t}$ is then given by $\rho_{\widehat{\varepsilon}}(1)=-0.5(\theta+1)$. The presence of conditional heteroscedasticity in the components of the random walk plus noise model can be identified analysing whether the autocorrelations of squared auxiliary residuals are larger than their squared autocorrelations.

The finite sample properties of the autocorrelations of $\left(\Delta y_{t}\right)^{2}, \widehat{\varepsilon}_{t}^{2}$ and $\widehat{\eta}_{t}^{2}$ are analyzed by means of extensive Monte Carlo experiments. The series have been generated with sample size $T=1000$ by the following four Q-STARCH models. 


\begin{tabular}{llllllllc} 
& $\alpha_{0}$ & $\alpha_{1}$ & $\alpha_{2}$ & $\beta$ & $\gamma_{0}$ & $\gamma_{1}$ & $\gamma_{2}$ & $\delta$ \\
\hline M1 & 0.25 & 0 & 0 & 0 & 0.05 & 0.15 & 0.8 & -0.17 \\
\hline M2 & 0.05 & 0.15 & 0.8 & -0.17 & 4.0 & 0 & 0 & 0 \\
\hline M3 & 4.0 & 0 & 0 & 0 & 0.05 & 0.15 & 0.8 & -0.17 \\
\hline M4 & 0.05 & 0.15 & 0.8 & -0.17 & 0.25 & 0 & 0 & 0 \\
\hline
\end{tabular}

The first two models have $q=4.0$, while $q=0.25$ for the other two. Models M1 and M3 have an homoscedastic short-run noise while the long-run component is heteroscedastic. On the other hand, the short-run disturbances of models M2 and M4 are heteroscedastic while the long-run variances are constant. The asymmetry parameter -0.17 has been chosen as it is the largest to guarantee the positivity of the conditional variances. Results for other designs and sample sizes are available from the authors upon request.

For each model, we generate 1000 replicates and for each replicate, we compute the sample autocorrelations of $\left(\Delta y_{t}\right)^{2}, \widehat{\varepsilon}_{t}^{2}$ and $\widehat{\eta}_{t}^{2}$ for lags up to 36 . Then, we compute the average mean and standard deviation of the estimates through all replicates. All simulations have been carried out on a Pentium desktop computer using our own FORTRAN codes.

The Monte Carlo results have been summarized in Fig. 1, that plots the mean autocorrelation function of $\left(\Delta y_{t}\right)^{2}$ together with the corresponding acf derived in previous section. In this figure, it can be observed that the biases are huge. Negative biases on the autocorrelations of squares of conditionally heteroscedastic series have been previously documented in the literature; see, for example Pérez and Ruiz (2003). On top of this, Fig. 1 illustrates that the biases are more severe when $q$ is large and the transitory component is conditionally heteroscedastic or when $q$ is small and the conditional heteroscedasticity appears in the long-run noise. In the first case, the marginal variance of the long-run component is larger than the marginal variance of the heteroscedastic transitory component. When computing the sample autocorrelations of squares these are heavily biased towards zero. A similar effect is observed when the long-run component is heterocedastic but its marginal variance is small when compared with the marginal variance of the transitory component. In these cases, it seems that the sample autocorrelations of $\left(\Delta y_{t}\right)^{2}$ are not useful to identify the presence of conditionally heteroscedastic unobserved noises.

The second and third rows of Fig. 1 plot the mean of the sample autocorrelations of the squared auxiliary residuals, $\widehat{\varepsilon}_{t}$ and $\widehat{\eta}_{t}$, together with the corresponding acf's obtained assuming homoscedasticity. First, notice that the autocorrelations are larger than expected if the corresponding component were homoscedastic. Therefore, the autocorrelations of squared auxiliary residuals can be a useful instrument to detect conditional heteroscedasticity. Furthermore, in the first two models, the autocorrelations of squares are larger in $\widehat{\eta}_{t}$ than in $\widehat{\varepsilon}_{t}$. On the other hand, for the last two models, the autocorrelations of $\widehat{\varepsilon}_{t}^{2}$ are larger than the autocorrelations of $\widehat{\eta}_{t}^{2}$. Notice that this is a rather useful result because it allows to identify the component that is conditionally heteroscedastic. Finally, it is also important to notice that, as expected, when the transitory noise, $\varepsilon_{t}$, is heteroscedastic, the autocorrelations of $\widehat{\varepsilon}_{t}^{2}$ are larger the smaller is $q$. However, when the conditional heteroscedasticity affects the long-run noise, $\eta_{t}$, the autocorrelations of $\widehat{\eta}_{t}^{2}$ are larger the larger is $q$. 

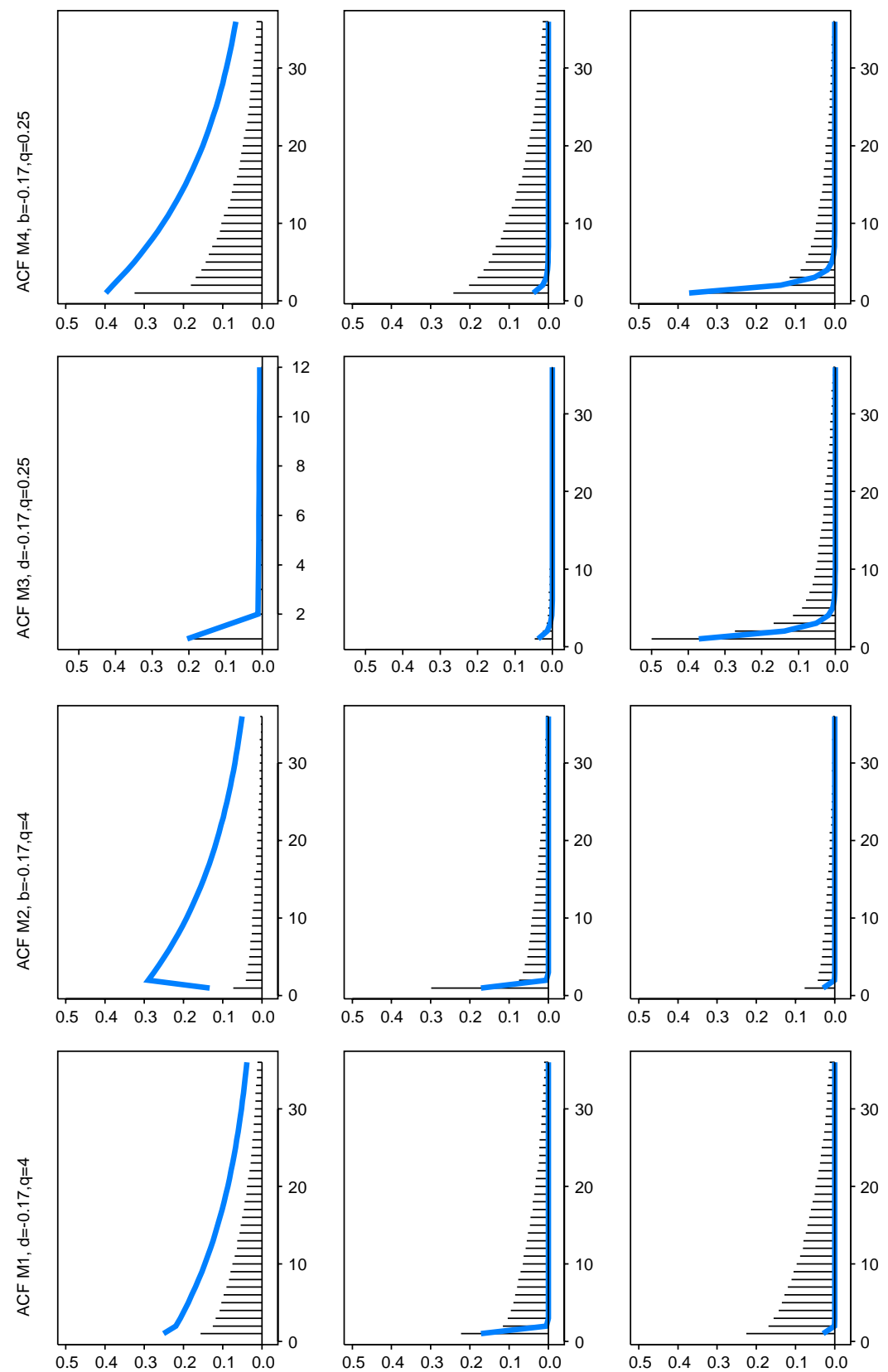

Fig. 1. Mean autocorrelation function of (by rows) $\left(\Delta y_{t}\right)^{2}, \hat{\varepsilon}_{t}^{2}$ and $\hat{\eta}_{t}^{2}$ for STARCH models (by columns). Results based on 1000 replications of series with sample size $T=1000$. 


\section{Estimation of Q-STARCH model}

There are several estimators proposed in the literature for the parameters of unobserved component models with heteroscedastic disturbances. For example, Dungey et al. (2000), Calzolari et al. (2004) and Sentana et al. (2004) propose to use indirect estimation methods. On the other hand, Fiorentini et al. (2004) have proposed an exact likelihood based estimator based on MCMC. In this paper, we focus on the QML estimator proposed by Harvey et al. (1992) because its simplicity and because it provides estimates of the latent components which are usually of interest in empirical applications.

The QML estimator of the parameters of the STARCH model is based on expressing the local level model in an augmented state space form. The state vector is augmented by lags of $\mu_{t}$ in such a way that it is possible to get estimations of both disturbances and their associated correction factors. The measurement and transition equations are, respectively, given by

$$
\begin{aligned}
& y_{t}=\mu_{t}+\varepsilon_{t}=\left[\begin{array}{lll}
1 & 0 & 0
\end{array}\right] \alpha_{t}+\varepsilon_{t}, \\
& \alpha_{t}=\left[\begin{array}{c}
\mu_{t} \\
\mu_{t-1} \\
\eta_{t}
\end{array}\right]=\left[\begin{array}{lll}
1 & 0 & 0 \\
1 & 0 & 0 \\
0 & 0 & 0
\end{array}\right]\left[\begin{array}{l}
\mu_{t-1} \\
\mu_{t-2} \\
\eta_{t-1}
\end{array}\right]+\left[\begin{array}{l}
1 \\
0 \\
1
\end{array}\right] \eta_{t} .
\end{aligned}
$$

Even if $\varepsilon_{t}^{\dagger}$ and $\eta_{t}^{\dagger}$ are assumed to be Gaussian processes, STARCH models are not conditionally Gaussian, since knowledge of past observations does not imply knowledge of past disturbances. Consequently, the QML estimator is based on treating the model as if it were conditionally Gaussian and running the Kalman filter to obtain the one-step ahead prediction errors and their variances to be used in the expression of the Gaussian likelihood given by

$$
\log L=-\frac{T}{2} \log (2 \pi)-\frac{1}{2} \sum_{t=1}^{T} \log F_{t}-\frac{1}{2} \sum_{t=1}^{T} \frac{v_{t}^{2}}{F_{t}},
$$

where $v_{t}, t=1, \ldots, T$ are the innovations and $F_{t}$ their corresponding variances. The QML estimator, $\widehat{\Psi}$, is obtained by maximizing the Gaussian likelihood in (14) with respect to the unknown parameters. Harvey et al. (1992) give a detailed description of the Kalman filter for the random walk plus noise model with GARCH disturbances.

Estimation of GQARCH models by QML is easier using the following reparametrization proposed by Sentana (1995) to guarantee the positivity of the variances $h_{t}$ and $q_{t}$,

$$
\begin{aligned}
& h_{t}=a_{0}+a_{1}^{2}\left(\varepsilon_{t-1}-b\right)^{2}+a_{2}^{2} h_{t-1}, \\
& q_{t}=g_{0}+g_{1}^{2}\left(\eta_{t-1}-d\right)^{2}+g_{2}^{2} q_{t-1},
\end{aligned}
$$

where the parameters of interest are $\alpha_{0}=a_{0}+a_{1}^{2} b^{2}, \alpha_{1}=a_{1}^{2}, \alpha_{2}=a_{2}^{2}$ and $\beta=-2 b a_{1}^{2}$. Similar transformations apply to the parameters of $q_{t}$. After estimating the parameter vector, $\Psi=\left(a_{0}, a_{1}, a_{2}, b, g_{0}, g_{1}, g_{2}, d\right)$, these transformations can be used to obtain the original parameters of the model. 
The Kalman filter for the Q-STARCH model requires expressions of the following estimates of $\varepsilon_{t}$ and $\eta_{t}$

$$
\begin{aligned}
& \tilde{\varepsilon}_{t}=y_{t}-m_{t}, \\
& \tilde{\eta}_{t}=m_{t}-m_{t-1 \mid t},
\end{aligned}
$$

where $m_{t}=E_{t} \mu_{t}$ and $m_{t-1 \mid t}=E_{t} \mu_{t-1}$ are MMSL updated estimates of $\mu_{t}$ and $\mu_{t-1}$ obtained in a natural way by the augmentation of the state vector by $\mu_{t-1}$ in (13). The $t$ under the expectation operator means that the expectation is conditional on the information available at time $t$. The filter also requires expressions of the conditional variances of the disturbances $\varepsilon_{t}$ and $\eta_{t}$. For simplicity, we consider first the Q-STARCH model with the parameters $\alpha_{2}$ and $\gamma_{2}$ fixed to zero. In this case, the conditional mean of $\varepsilon_{t}$ is zero and its conditional variance is given by

$$
H_{t}=\underset{t-1}{E} \varepsilon_{t}^{2}=a_{0}+a_{1}^{2}\left(\tilde{\varepsilon}_{t-1}-b\right)^{2}+a_{1}^{2} P_{t-1},
$$

where $P_{t}=E_{t}\left(\mu_{t}-m_{t}\right)$. Similarly, the conditional mean of the disturbance of the permanent component, $\eta_{t}$, is zero and its conditional variance is given by

$$
Q_{t}=\underset{t-1}{E} \eta_{t}^{2}=g_{0}+g_{1}^{2}\left(\tilde{\eta}_{t-1}-d\right)^{2}+g_{1}^{2} P_{t-1}^{\eta},
$$

where $P_{t-1}^{\eta}=P_{t}+P_{t-1 \mid t}-2 P_{t, t-1 \mid t}, P_{t-1 \mid t}=E_{t}\left(\mu_{t-1}-m_{t-1 / t}\right)^{2}$ and $P_{t, t-1 / t}=E_{t}\left(\mu_{t}-m_{t}\right)$ $\left(\mu_{t-1}-m_{t-1 / t}\right)$. The required $P_{t}, P_{t-1 \mid t}$ and $P_{t, t-1 \mid t}$ are also provided by the Kalman filter. In order to carry out the initialization of the filter, we set $m_{1}=y_{1}$ and $P_{1}=E_{0} \varepsilon_{1}=$ $\sigma_{\varepsilon}^{2}=\left(a_{0}+a_{1}^{2} b^{2}\right) /\left(1-a_{1}^{2}\right)$. In the framework of a random walk plus white noise this is equivalent to use a diffuse prior. Furthermore, if the conditional variance of $\eta_{t}$ at time $t-1$ is also set equal to its unconditional variance, the Kalman filter can be started with $E_{t-1}\left(\varepsilon_{2}^{2}\right)=\sigma_{\varepsilon}^{2}$ and $E_{t-1}\left(\eta_{2}^{2}\right)=\sigma_{\eta}^{2}$.

If the parameters $\alpha_{2}$ and $\gamma_{2}$ are different from zero, Harvey et al. (1992) suggest to consider the following alternative definitions of $h_{t}$ and $q_{t}$ :

$$
\begin{aligned}
& h_{t}=a_{0}+a_{1}^{2}\left(\varepsilon_{t-1}-b\right)^{2}+a_{2}^{2} \underset{t-2}{E}\left(h_{t-1}\right), \\
& q_{t}=g_{0}+g_{1}^{2}\left(\eta_{t-1}-d\right)^{2}+g_{2}^{2} \underset{t-2}{E}\left(q_{t-1}\right) .
\end{aligned}
$$

Notice that $E_{t-1}\left(\varepsilon_{t}^{2}\right)=E_{t-1}\left(h_{t}\right)$ and $E_{t-1}\left(\eta_{t}^{2}\right)=E_{t-1}\left(q_{t}\right)$. Consequently, using Eqs. (17) and (18), the following expressions are obtained:

$$
\begin{aligned}
& H_{t}=a_{0}+a_{1}^{2}\left(\tilde{\varepsilon}_{t-1}-b\right)^{2}+a_{1}^{2} P_{t-1}+a_{2}^{2} H_{t-1}, \\
& Q_{t}=g_{0}+g_{1}^{2}\left(\tilde{\eta}_{t-1}-d\right)^{2}+g_{1}^{2} P_{t-1}^{\eta}+g_{2}^{2} Q_{t-1} .
\end{aligned}
$$

In order to obtain the asymptotic distribution of the QML estimator, Harvey et al. (1992) suggest to consider that the variances $h_{t}$ and $q_{t}$ are given by Eqs. (20). In this case, the Kalman filter is exactly the same as the one previously described but the model is conditionally Gaussian. Consequently, the filter and the likelihood in (14) are exact and the 
usual asymptotic theory can be applied. Under very general conditions, the asymptotic distribution of $\widehat{\Psi}$ can be approximated by a multivariate normal distribution with mean $\Psi$ and covariance matrix $(A v a r)^{-1}$. The $i j^{\prime}$ th element of the matrix Avar is given by

$$
I A_{i j}(\Psi)=\frac{1}{2} E\left[\sum_{t=1}^{T} \frac{1}{F_{t}^{2}} \frac{\partial F_{t}}{\partial \Psi} \frac{\partial F_{t}}{\partial \Psi^{\prime}}+\sum_{t=1}^{T} \frac{1}{F_{t}} \frac{\partial v_{t}}{\partial \Psi} \frac{\partial v_{t}}{\partial \Psi^{\prime}}\right]
$$

The derivatives in expression (21) can be numerically evaluated as explained by Harvey (1989). Once, the matrix Avar has been computed, the delta method can be used to obtain the covariance matrix of the parameters of interest.

The finite sample properties of the QML estimator are analyzed by means of Monte Carlo experiments. The series are simulated by the following alternative Q-STARCH models with parameters $\left(\alpha_{0}, \alpha_{1}, \alpha_{2}, \beta, \gamma_{0}, \gamma_{1}, \gamma_{2}, \delta\right)$ given by

\begin{tabular}{lllllllll} 
& $\alpha_{0}$ & $\alpha_{1}$ & $\alpha_{2}$ & $\beta$ & $\gamma_{0}$ & $\gamma_{1}$ & $\gamma_{2}$ & $\delta$ \\
\hline M1 & 0.01 & 0.2 & 0 & -0.05 & 0.01 & 0.1 & 0 & -0.05 \\
\hline M2 & 0.01 & 0.2 & 0.5 & -0.05 & 0.01 & 0.1 & 0.7 & -0.05 \\
\hline M3 & 0.25 & 0 & 0 & 0 & 0.05 & 0.15 & 0.8 & 0 \\
\hline M4 & 0.05 & 0.15 & 0.8 & 0 & 4 & 0 & 0 & 0 \\
\hline
\end{tabular}

In model M1 both components are heteroscedastic with ARCH disturbances while in model M2, the disturbances are GARCH. The next two models have been chosen because the asymmetric parameters $\beta$ and $\delta$ are zero so we can analyze whether the sample distribution of the QML estimators of these parameters can be used to infer whether the transitory or the long-run conditional variances are asymmetric. Results for other parameter designs are available by the authors upon request.

The sample sizes considered are $T=300,1000$ and 3000. The numerical optimization of the likelihood has been performed using the IMSL subroutine DBCPOL with the parameters $\alpha_{0}$ and $\gamma_{0}$ restricted to be nonnegative, and $\alpha_{1}+\alpha_{2}$ and $\gamma_{1}+\gamma_{2}$ restricted to be between 0 and 1.

Table 1 reports the Monte Carlo means and standard deviations (brackets) for models M1 and M2. This table also shows, in squared brackets, the corresponding approximated asymptotic standard deviation computed using expression (20). The results for model M1 show that, the biases of all the parameters are rather small even when $T=300$. However, the asymptotic standard deviations of the $\mathrm{ARCH}$ parameters provide an adequate approximation to the empirical standard deviations only for the biggest sample size. In general, the asymptotic standard deviation is larger than the empirical standard deviation that decreases with the sample size at rate $\sqrt{T}$, approximately. Fig. 2 plots kernel estimates of the densities of the parameter estimates of this model. This figure illustrates that the asymptotic Normal approximation of the QML estimator is only adequate for relatively large sample sizes as, for example, $T=3000$. 
Table 1

Monte Carlo results for estimated parameters of Q-STARCH models with asymmetry in both components. Standard deviations in brackets. Asymptotic Standard deviations in squared brackets

\begin{tabular}{|c|c|c|c|c|c|c|c|}
\hline & \multicolumn{3}{|l|}{$T$} & & \multicolumn{3}{|l|}{$T$} \\
\hline & 300 & 1000 & 3000 & & 300 & 1000 & 3000 \\
\hline$\alpha_{0}=0.01$ & $\begin{array}{c}0.0101 \\
(0.0023) \\
{[0.0030]}\end{array}$ & $\begin{array}{c}0.0100 \\
(0.0011) \\
{[0.0016]}\end{array}$ & $\begin{array}{c}0.0100 \\
(0.0007) \\
{[0.0009]}\end{array}$ & $\alpha_{0}=0.01$ & $\begin{array}{c}0.0112 \\
(0.0101) \\
{[0.0243]}\end{array}$ & $\begin{array}{c}0.0110 \\
(0.0080) \\
{[0.0128]}\end{array}$ & $\begin{array}{c}0.0101 \\
(0.0057) \\
{[0.0071]}\end{array}$ \\
\hline$\alpha_{1}=0.2$ & $\begin{array}{c}0.2084 \\
(0.1328) \\
{[0.2036]}\end{array}$ & $\begin{array}{c}0.2087 \\
(0.0563) \\
{[0.1037]}\end{array}$ & $\begin{array}{c}0.2139 \\
(0.0378) \\
{[0.0582]}\end{array}$ & $\alpha_{1}=0.2$ & $\begin{array}{c}0.3158 \\
(0.2631) \\
{[0.6679]}\end{array}$ & $\begin{array}{c}0.2968 \\
(0.2282) \\
{[0.3706]}\end{array}$ & $\begin{array}{c}0.2822 \\
(0.1712) \\
{[0.2093]}\end{array}$ \\
\hline$\alpha_{2}=0.0$ & & & & $\alpha_{2}=0.5$ & $\begin{array}{c}0.3647 \\
(0.3171) \\
{[1.1964]}\end{array}$ & $\begin{array}{c}0.3815 \\
(0.2894) \\
{[0.6342]}\end{array}$ & $\begin{array}{c}0.4220 \\
(0.2517) \\
{[0.3518]}\end{array}$ \\
\hline$\beta=-0.05$ & $\begin{array}{r}-0.0433 \\
(0.0246) \\
{[0.0300]}\end{array}$ & $\begin{array}{r}-0.0464 \\
(0.0122) \\
{[0.0143]}\end{array}$ & $\begin{array}{r}-0.0480 \\
(0.0063) \\
{[0.0085]}\end{array}$ & $\beta=-0.05$ & $\begin{array}{r}-0.0354 \\
(0.0676) \\
{[0.0995]}\end{array}$ & $\begin{array}{r}-0.0340 \\
(0.0471) \\
{[0.0509]}\end{array}$ & $\begin{array}{r}-0.0315 \\
(0.0270) \\
{[0.0284]}\end{array}$ \\
\hline$\gamma_{0}=0.01$ & $\begin{array}{c}0.0096 \\
(0.0020) \\
{[0.0035]}\end{array}$ & $\begin{array}{c}0.0096 \\
(0.0011) \\
{[0.0019]}\end{array}$ & $\begin{array}{c}0.0096 \\
(0.0006) \\
{[0.0011]}\end{array}$ & $\gamma_{0}=0.01$ & $\begin{array}{c}0.0167 \\
(0.0165) \\
{[0.0087]}\end{array}$ & $\begin{array}{c}0.0121 \\
(0.0076) \\
{[0.0044]}\end{array}$ & $\begin{array}{c}0.0106 \\
(0.0034) \\
{[0.0026]}\end{array}$ \\
\hline$\gamma_{1}=0.1$ & $\begin{array}{c}0.0988 \\
(0.0464) \\
{[0.2737]}\end{array}$ & $\begin{array}{c}0.1045 \\
(0.0319) \\
{[0.1483]}\end{array}$ & $\begin{array}{c}0.1082 \\
(0.0188) \\
{[0.0849]}\end{array}$ & $\gamma_{1}=0.2$ & $\begin{array}{c}0.2732 \\
(0.1782) \\
{[0.1351]}\end{array}$ & $\begin{array}{c}0.2684 \\
(0.1065) \\
{[0.0727]}\end{array}$ & $\begin{array}{c}0.2728 \\
(0.0591) \\
{[0.0451]}\end{array}$ \\
\hline$\gamma_{2}=0.0$ & & & & $\gamma_{2}=0.7$ & $\begin{array}{c}0.5365 \\
(0.2585) \\
{[0.1970]}\end{array}$ & $\begin{array}{c}0.6042 \\
(0.1503) \\
{[0.0986]}\end{array}$ & $\begin{array}{c}0.6190 \\
(0.0771) \\
{[0.0596]}\end{array}$ \\
\hline$\delta=-0.05$ & $\begin{array}{r}-0.0426 \\
(0.0177) \\
{[0.0383]}\end{array}$ & $\begin{array}{r}-0.0469 \\
(0.0076) \\
{[0.0193]}\end{array}$ & $\begin{array}{r}-0.0486 \\
(0.0033) \\
{[0.0112]}\end{array}$ & $\delta=-0.05$ & $\begin{array}{r}-0.0605 \\
(0.0543) \\
{[0.0495]}\end{array}$ & $\begin{array}{r}-0.0565 \\
(0.0285) \\
{[0.0247]}\end{array}$ & $\begin{array}{r}-0.0564 \\
(0.0149) \\
{[0.0137]}\end{array}$ \\
\hline
\end{tabular}

The results for model M2 are, in general, similar to the previous ones. However, it is possible to observe that it seems to be a negative correlation between the parameters $\alpha_{1}$ and $\alpha_{2}$. The parameter $\alpha_{1}$ is overestimated while $\alpha_{2}$ is underestimated. The same effect can be observed with respect to the parameters $\gamma_{1}$ and $\gamma_{2}$. For example, when $T=300$, the empirical correlations between $\alpha_{1}$ and $\alpha_{2}$ and between $\gamma_{1}$ and $\gamma_{2}$ are -0.57 and -0.61 , respectively. When the sample size is $T=3000$, these correlations are even bigger, -0.73 and -0.88 respectively. Notice that these high correlations could be expected since we are estimating imposing the stationarity restrictions, $\alpha_{1}+\alpha_{2}<1$ and $\gamma_{1}+\gamma_{2}<1$ and the parameters chosen are very close to these boundaries. On top of that, 

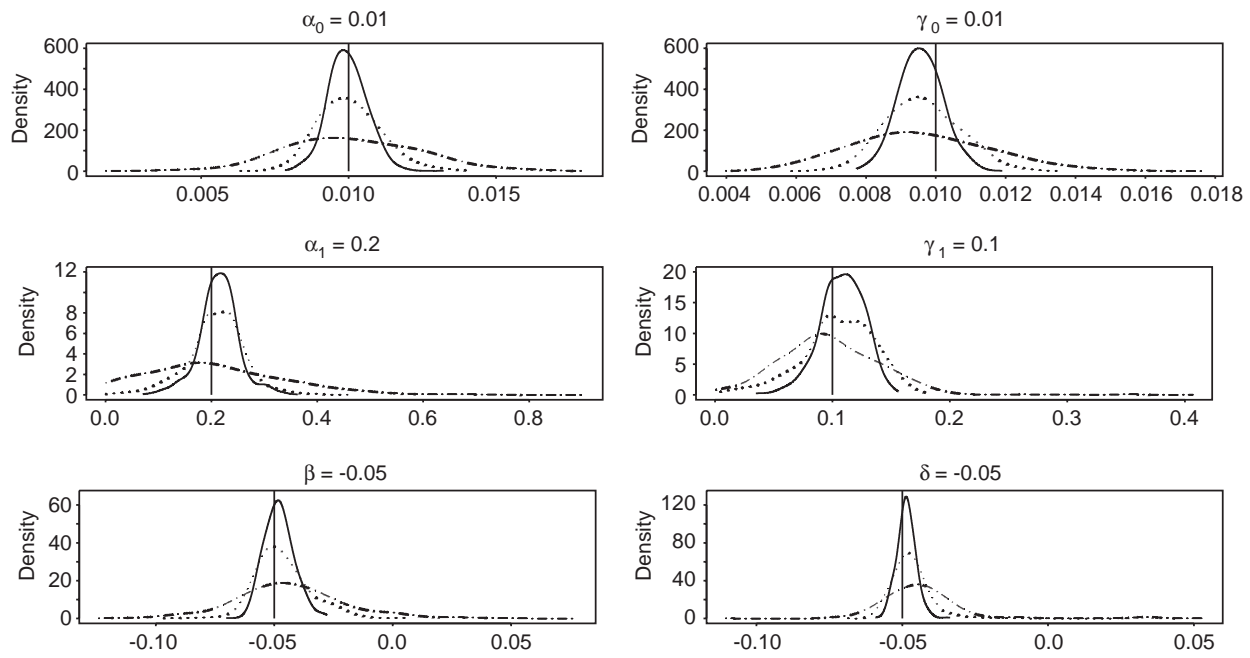

Fig. 2. Kernel densities of the QML estimates of the estimated parameters of a Q-STARCH model with $\alpha_{0}=0.01$, $\gamma_{0}=0.01, \alpha_{1}=0.2, \gamma_{1}=0.1, \beta=-0.05$ and $\delta=-0.05$. The dash-dotted line corresponds to $T=300$, the dotted line to $T=1000$ and the solid line to $T=3000$.

we can observe that the presence of the GARCH parameters worsens the estimation of the asymmetry parameter, specially if such asymmetry appears in the short-run variance.

To illustrate the problems faced when the Quasi-likelihood is maximized, Fig. 3 plots the Gaussian likelihood in (14) for series simulated by Q-STARCH processes with asymmetry and conditional heteroscedasticity in the transitory component and four different specifications in the permanent one as a function of the parameters $a_{1}$ and $b$. Note that the function becomes flatter as the number of parameters increases. On the other hand, Fig. 3 shows that the log-likelihood has local maximum, and consequently, the performance of any optimization algorithm strongly depends on the initial values provided. Finally, it is important to realize that the difficulties estimating the parameter $\alpha_{1}=a_{1}^{2}$ could be due to the fact that the $\log$-likelihood is rather flat when $b$ is in its maximum.

Finally, Fig. 4 plots kernel estimates of the densities of the Monte Carlo of the estimates of the parameters $\beta$ and $\delta$ of models M3 and M4, which are actually zero. Looking at these kernel densities, it seems that the null hypothesis $\mathrm{H}_{0}: \beta=0$ can be tested using standard results.

\section{Empirical application}

In this section we fit the Q-STARCH model to two daily financial series of returns, a daily series of gold prices and two monthly inflation series.

The two financial time series are daily prices of the Nikkei 225 index observed from January 3, 1994 to December, 29, 2000 with $T=1825$ and of the Hewlett-Packard stock 

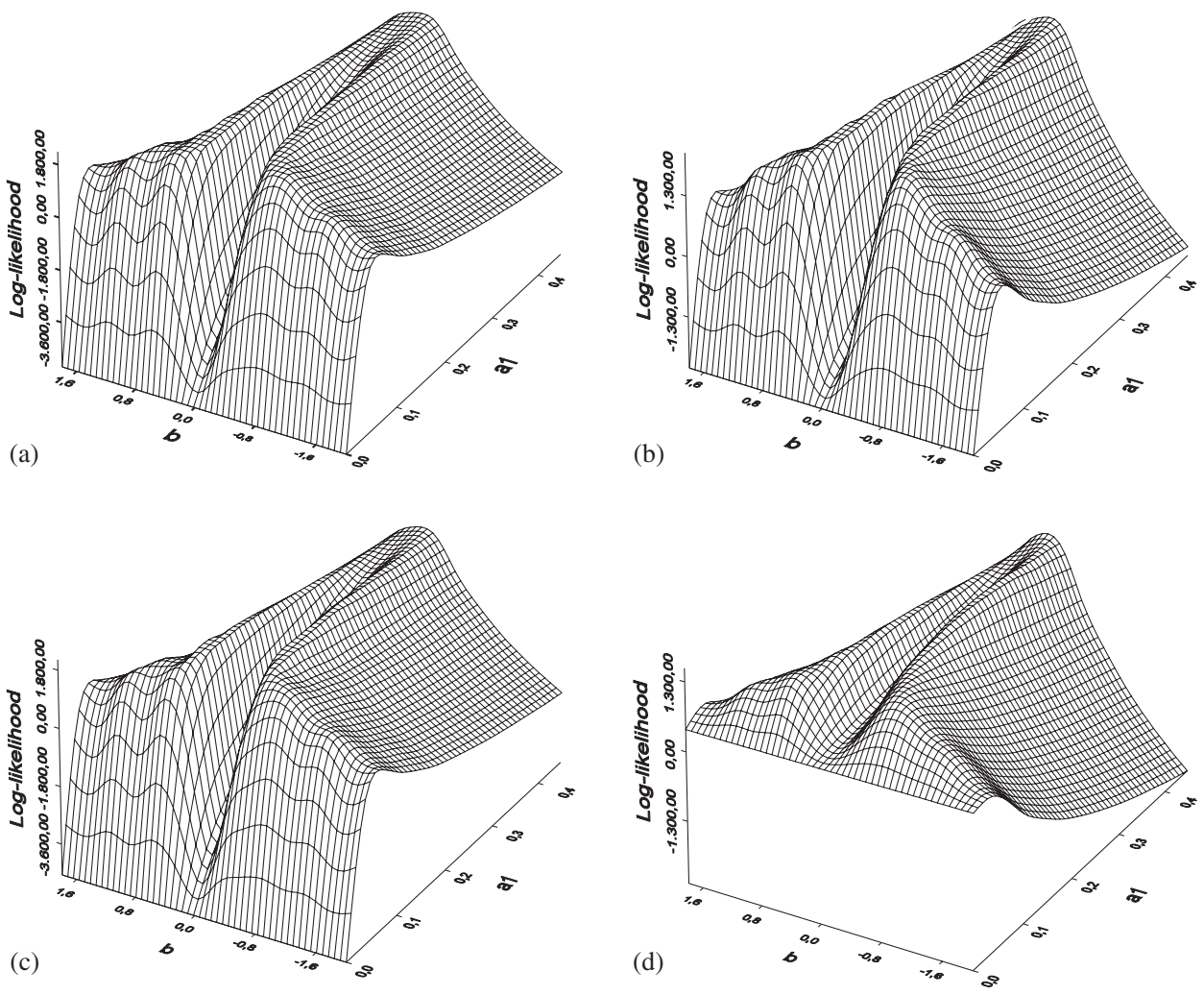

Fig. 3. Likelihood with respect to $a_{1}$ and $b$ when (a) $\eta_{t}$ is homoscedastic, (b) $\eta_{t}$ is an ARCH(1) process, (c) $\eta_{t}$ is an QARCH process and (d) $\eta_{t}$ is an GQARCH $(1,1)$ process.
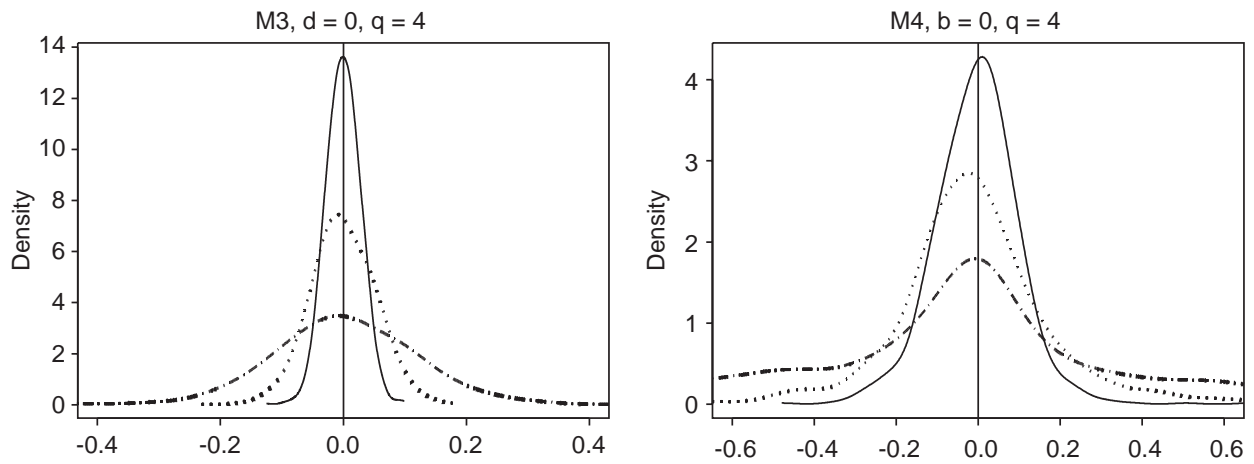

Fig. 4. Kernel densities for the estimated asymmetry parameters in Q-STARCH models. The dash-dotted line corresponds to $T=300$, the dotted line to $T=1000$ and the solid line to $T=3000$. 
Table 2

Summary statistics of $\Delta y_{t}$ for Nikkei 225, Hewlett-Packard, gold prices series and Japan and United Kingdom inflation series

\begin{tabular}{|c|c|c|c|c|c|c|c|c|c|c|}
\hline & & \multicolumn{2}{|l|}{ NIK } & \multicolumn{2}{|l|}{ HPQ } & \multicolumn{2}{|c|}{ GOLD } & \multicolumn{2}{|l|}{ JAP } & UK \\
\hline Mean & & \multicolumn{2}{|l|}{-0.012} & \multicolumn{2}{|c|}{0.025} & \multicolumn{2}{|l|}{0.000} & \multicolumn{2}{|c|}{0.000} & 0.000 \\
\hline SK & & \multicolumn{2}{|l|}{0.013} & \multicolumn{2}{|c|}{-0.062} & \multicolumn{2}{|l|}{0.698} & \multicolumn{2}{|c|}{-0.055} & 0.117 \\
\hline$\kappa$ & & \multicolumn{2}{|l|}{5.588} & \multicolumn{2}{|c|}{6.920} & \multicolumn{2}{|l|}{9.159} & \multicolumn{2}{|c|}{3.985} & 3.624 \\
\hline$\rho(\tau)$ & $\Delta y_{t}$ & $\left(\Delta y_{t}\right)^{2}$ & $\Delta y_{t}$ & $\left(\Delta y_{t}\right)^{2}$ & $\Delta y_{t}$ & $\left(\Delta y_{t}\right)^{2}$ & $\Delta y_{t}$ & $\left(\Delta y_{t}\right)^{2}$ & $\Delta y_{t}$ & $\left(\Delta y_{t}\right)^{2}$ \\
\hline 1 & $-0.045^{*}$ & $0.059^{*}$ & -0.017 & $0.048^{*}$ & $-0.096^{*}$ & $0.054^{*}$ & $-0.549^{*}$ & $0.384^{*}$ & $-0.376^{*}$ & $0.211^{*}$ \\
\hline 2 & -0.010 & $0.091^{*}$ & $-0.057^{*}$ & $0.059^{*}$ & 0.020 & $0.036^{*}$ & 0.035 & $0.133^{*}$ & $-0.115^{*}$ & 0.028 \\
\hline 3 & $-0.029 *$ & $0.048^{*}$ & -0.018 & $0.038^{*}$ & 0.021 & $0.044^{*}$ & $0.075^{*}$ & $0.064^{*}$ & $0.107^{*}$ & 0.043 \\
\hline 4 & $0.032 *$ & $0.129^{*}$ & 0.011 & $0.047^{*}$ & -0.023 & 0.022 & $-0.098^{*}$ & $0.131^{*}$ & $-0.105^{*}$ & $-0.068^{*}$ \\
\hline 5 & -0.024 & $0.101^{*}$ & -0.018 & $0.071^{*}$ & $-0.042^{*}$ & $0.062^{*}$ & $0.067^{*}$ & $0.182^{*}$ & 0.006 & 0.006 \\
\hline$Q(10)$ & 18.982 & $127.27^{*}$ & 17.017 & $76.406^{*}$ & 16.224 & 20.172 & $134.480^{*}$ & $114.040^{*}$ & $93.756^{*}$ & $27.164^{*}$ \\
\hline
\end{tabular}

* Significant at the $5 \%$ level.

observed from January 3, 1994 to May 20, 2003 with $T=2362$. We also analyze a daily series of the logarithm of gold prices in US observed from January 1, 1985 to December, 3, 1989 with $T=1074$. Several descriptive sample moments of the first differences of these series are reported in Table 2.All the series show excess kurtosis and autocorrelations of squares larger than expected if they were linear. The two inflation series are monthly observations corresponding to Japan and United Kingdom. Inflation rates, $y_{t}$, are obtained as $y_{t}=\left(\log \left(\mathrm{CPI}_{t}\right)-\log \left(\mathrm{CPI}_{t-1}\right)\right) \times 100$ where CPI stands for consumer price index. The UK CPI was observed from January, 1962 to August, 2001 with $T=476$, while for Japan the data were observed from January, 1970 to August, 2001, with $T=380$. Intervention analysis and seasonal adjustment of inflation series were carried out with the program STAMP 6.20. Several descriptive sample moments of $\Delta y_{t}$ are reported in Table 2. In these series the evidence of conditional heteroscedasticity is not so strong as in the daily series analyzed before.

The estimates of the parameters of the homoscedastic random walk plus noise model for the five series analyzed are shown in Table 3. These estimates have been obtained using the program STAMP 6.20 of Koopman et al. (2000). Note that looking at the results for the financial and gold prices it is possible to observe that the estimated signal to noise ratio, $\hat{q}$, is rather large, meaning that in these series the variability of the permanent component dominates. However, in the two inflation series, $\widehat{q}$, is less than one, meaning that the estimated variance of the permanent component, $\hat{\sigma}_{\eta}^{2}$, is small compared with the variance of the transitory component, $\hat{\sigma}_{\varepsilon}^{2}$.

Table 3 reports several diagnostic statistics of the estimated innovations, $v_{t}$, and the auxiliary residuals, $\hat{\varepsilon}_{t}$ and $\hat{\eta}_{t}$. In particular, for each of these series, we report the Box-Ljung statistics of order $10, Q(10)$, for the original series and their squares. With respect to the innovations, $Q(10)$ does not show evidence of autocorrelation in any of the series. However, the corresponding statistic for the squares, $Q_{2}(10)$, is highly significant at any usual level. Consequently, the series of innovations may exhibit some kind of conditional 
Table 3

QML estimates of the parameters of the random walk plus noise model and summary statistics of estimated innovations and auxiliary residuals

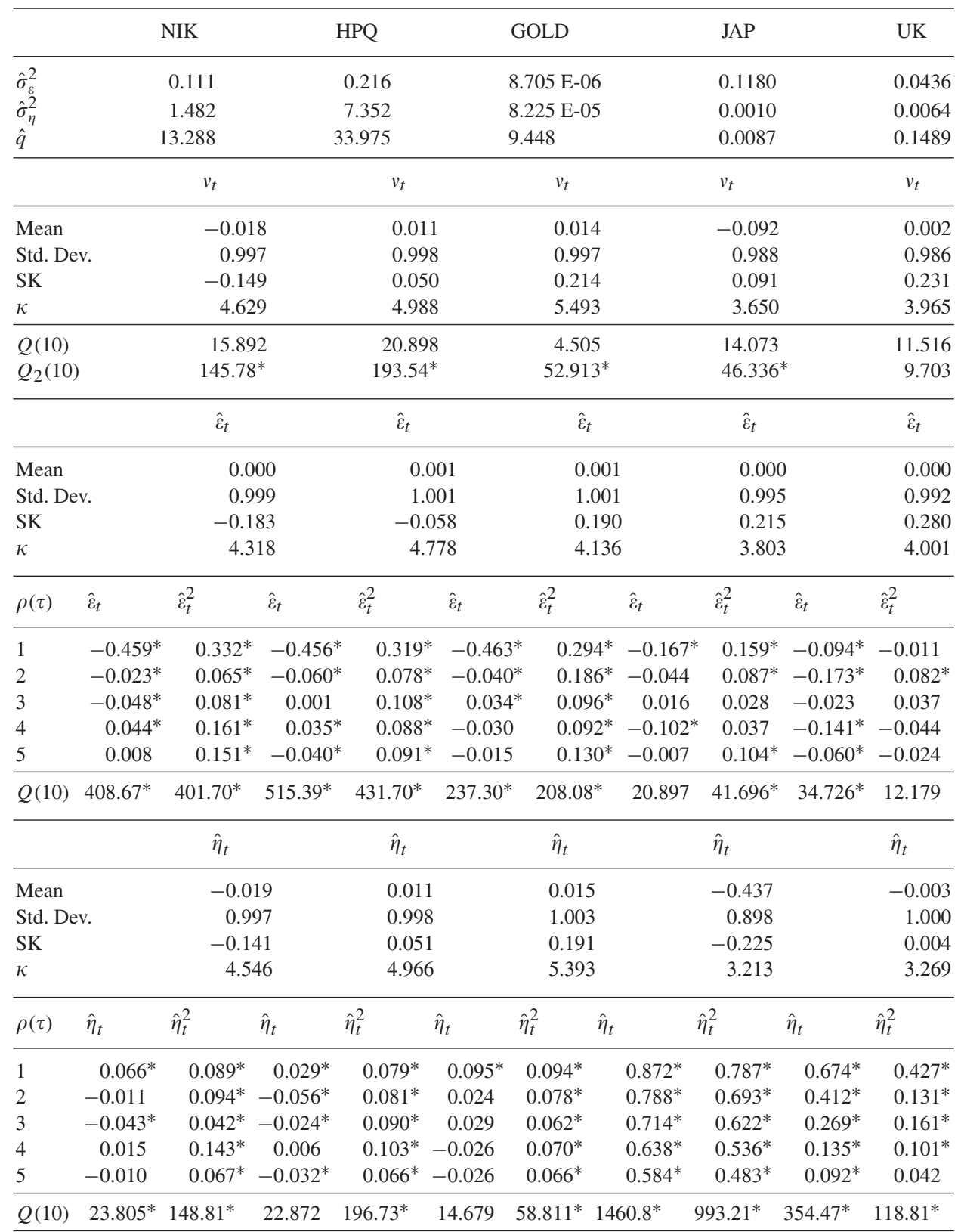

* Significant at the $5 \%$ level. 
heteroscedasticity. With respect to the auxiliary residuals, remember that they are serially correlated. For instance, the theoretical autocorrelation of order one for the Nikkei of $\widehat{\varepsilon}_{t}$ is $\rho_{\varepsilon}(1)=-0.4671$ and the theoretical acf of $\widehat{\eta}_{t}$ is $\rho_{\eta}(\tau)=0.066 \rho(\tau-1), \tau=2,3, \ldots$, with $\rho_{\eta}(1)=0.66$. Observe that the estimated autocorrelations in Table 3 are very close to their theoretical counterparts. If the noises were homoscedastic, the autocorrelations of the squared residuals are expected to be equal to the squared autocorrelations of the original residuals.

Looking first at the results for the financial and gold prices, it is possible to observe that the autocorrelations of $\widehat{\eta}_{t}^{2}$ are clearly larger than the squared autocorrelations. Furthermore, notice that the Box-Ljung statistic of squares is much larger than the corresponding statistic for $\widehat{\eta}_{t}$. However, when looking at the autocorrelations of the auxiliary residuals of the transitory component, the Box-Ljung statistic of $\widehat{\varepsilon}_{t}^{2}$ is smaller than the corresponding statistic for $\widehat{\varepsilon}_{t}$. Consequently, the analysis of the diagnostics in Table 3 seems to suggest that in these prices, the long-run component is heteroscedastic while the transitory component is homoscedastic.

Therefore, we fit the following Q-STARCH model to these series:

$$
\begin{array}{ll}
y_{t}=\mu_{t}+\varepsilon_{t}, & \operatorname{Var}\left(\varepsilon_{t}\right)=\alpha_{0}, \\
\mu_{t}=\mu_{t-1}+\eta_{t}, & \operatorname{Var}\left(\eta_{t}\right)=\gamma_{0}+\gamma_{1} \eta_{t-1}^{2}+\delta \eta_{t-1}+\gamma_{2} q_{t-1} .
\end{array}
$$

The QML estimates of the parameters are

NIK: $\quad h_{t}=0.079$;

$$
q_{t}=\underset{(3.468)}{0.035}+\underset{(5.082)}{0.082} \eta_{t-1}^{2}+\underset{(51.752)}{0.900} q_{t}^{2}-\underset{(4.949)}{0.108} \eta_{t-1}
$$

HPQ: $\quad h_{t}=0.399$;

$$
q_{t}=\underset{(2.896)}{0.033}+\underset{(4.886)}{0.021} \eta_{t-1}^{2}+\underset{(193.257)}{0.973} q_{t}^{2}-\underset{(2.274)}{0.053} \eta_{t-1},
$$

GOLD: $\quad h_{t}=1.0 E-05$

$$
q_{t}=\underset{(2.032)}{4.0 E-06}+\underset{(2.869)}{0.054} \eta_{t-1}^{2}+\underset{(28.265)}{0.897} q_{t}^{2}+\underset{(3.071)}{0.001} \eta_{t-1}
$$

The values between brackets are $t$-statistics. The ARCH effects are significant for the three series considered. Therefore, these series have an underlying level that is heteroscedastic and a homoscedastic short-run component. Furthermore, the estimate of the asymmetry parameter, $\delta$, is significant and negative for the two financial prices considered. Therefore, our results are in concordance with the stylized fact, frequently observed in the empirical analysis of financial returns, that the response of the volatility is larger when the returns are negative than when they are positive. However, notice that the Q-STARCH model allows 
us to conclude that this effect appears in the long-run component of prices and not in the transitory component. Obviously, the returns series inheritate the leverage effect.

Finally, notice that the asymmetric effect of the gold prices is positive. Therefore, increases in the permanent component have a larger effect on future uncertainty of prices than decreases on the long-run component. As in the case of the financial prices, the transitory component is homoscedastic.

Looking now at the diagnostics reported in Table 3 for the two inflation series, it is possible to observe that the innovations corresponding to Japan show some kind of conditional heteroscedasticity while in UK the autocorrelations of squared innovations are not significant. The same conclusion is reached looking at the Box-Ljung statistics for the squared residuals of the transitory component. Finally, notice that the autocorrelations of $\hat{\eta}_{t}^{2}$ are approximately equal to the squared autocorrelations of $\hat{\eta}_{t}$. Therefore, it seems that the longrun noises are not conditionally heteroscedastic while the transitory noises of Japan may have some kind of conditional heteroscedasticity.

In this case, the preferred Q-STARCH model consists in a $\operatorname{GQARCH}(1,1)$ model for the transitory component, and no ARCH effect in the permanent component disturbance given by

$$
\begin{array}{ll}
y_{t}=\mu_{t}+\varepsilon_{t}, & \operatorname{Var}\left(\varepsilon_{t}\right)=\alpha_{0}+\alpha_{1} \varepsilon_{t-1}^{2}+\beta \varepsilon_{t-1}+\alpha_{2} h_{t-1}, \\
\mu_{t}=\mu_{t-1}+\eta_{t}, & \operatorname{Var}\left(\eta_{t}\right)=\gamma_{0} .
\end{array}
$$

The QML estimates of the parameters are

$$
\begin{aligned}
\mathrm{JAP}: \quad h_{t}= & \underset{(2.043)}{0.006}+\underset{(3.319)}{0.187} \varepsilon_{t-1}^{2}+\underset{(12.589)}{0.784} h_{t}^{2}+\underset{(1.542)}{0.029} \varepsilon_{t-1} ; \\
q_{t}= & \begin{aligned}
0.001, \\
(1.999)
\end{aligned} \\
\mathrm{UK}: \quad h_{t}= & \underset{(0.0 E-04}{ }(0.913) \\
q_{t}= & 0.003 .
\end{aligned}
$$

The estimation results are in concordance with the conclusions derived from the analysis of the auxiliary residuals. The ARCH parameter $\alpha_{1}$ is clearly significant for Japan while for UK is not statistically different from zero. Therefore, the monthly inflation in UK seems to be homoscedastic, while in Japan the short-run component is conditionally heteroscedastic. However, the asymmetry parameter is significant in Japan at the $10 \%$ level. As this parameter is positive, it implies that when the short-run inflation rises, the uncertainty associated with future inflation increases more than when it goes down. On the other hand, according to the estimated Q-STARCH models, the underlying long-run inflation is homoscedastic. Finally, notice that these estimates support the Friedman hypothesis, according to which, a positive shock in inflation affects future uncertainty about inflation more than a negative one; see Friedman (1977). Previous results on testing the Friedman hypothesis are rather contradictory because the models proposed were not in general able to differentiate 
whether the long and short-run components were homoscedastic with asymmetric responses of the uncertainty. The Q-STARCH model proposed in this paper can help to separate these effects.

\section{Summary and conclusions}

In this paper we propose a new unobserved components model with conditionally heteroscedastic noises that allows the corresponding conditional variances to respond asymmetrically to negative and positive shocks. We denote this model as Q-STARCH. We show that the asymptotic distribution of a QML estimator could be an adequate approximation to the finite sample distribution. Consequently, inference can be based on classical results.

We also show how the autocorrelations of squared auxiliary residuals contain information useful to identify which of the components is conditionally heteroscedastic. However, the sample autocorrelations are severely biased towards zero making, in some cases, the identification of conditional heteroscedasticity a difficult task. In this sense, it may be useful to analyze the behavior of the portmanteau statistic proposed by Rodriguez and Ruiz (2003) to test the uncorrelatedness of a time series that takes into account not only the magnitude of the sample autocorrelations but also whether these autocorrelations have any systematic pattern.

Finally, we show with empirical examples how the Q-STARCH model can be useful for both financial and macroeconomic variables.

Two generalizations of the model are of special interest for the empirical applications: first, the extension to models with seasonal components so that the model can be directly implemented to analyze seasonal data as inflation, and second, the multivariate generalization. Further research is being carried out in these directions.

\section{Acknowledgements}

Financial support from projects IHP-RNT-00-2 by the European program "Research Training Networks" and BEC2002.03720 from the Spanish Government are gratefully acknowledge. We are grateful for very useful comments from three anonimous referees, the editor Stephen Pollock and participants at a seminar at Timbergen Institute. The usual disclaimers apply.

\section{References}

Ball, L., Cecchetti, S.G., 1990. Inflation and uncertainty at short and long horizons. Brooking Papers on Economic Activity, 215-254.

Bos, C., Mahieu, R., van Dijk, H., 2000. On the variation of hedging decisions in daily currency risk management. Econometric Institute Report EI 2000-20/A, Erasmus University, Rotterdam. 
Breidt, F.J., Davis, R.A., 1992. Time-reversibility, identifiability and independence of innovations for stationary time series. Journal of Time Series Analysis 13, 377-390.

Brunner, A.D., Hess, G.D., 1993. Are higher levels of inflation less predictable? A state dependent conditional heteroskedasticity approach. Journal of Business and Economic Statistics 11, 187-197.

Calzolari, G., Fiorentini, G., Sentana, E., 2004. Constrained indirect estimation. Review of Economic Studies, 71, 945-973.

Diebold, F.X., Nerlove, M., 1989. The dynamic of exchange rate volatility: A multivariate latent factor ARCH model. Journal of Applied Econometrics 4, 1-21.

Dungey, M., Martin, V.L., Pagan, A.R., 2000. A multivariate latent factor decomposition of international bond yield spreads. Journal of Applied Econometrics 15, 697-715.

Durbin, J., Koopman, S.J., 2001. Time Series by State Space Methods. Oxford University Press, Oxford.

Evans, M., 1991. Discovering the link between inflation rates and inflation uncertainty. Journal of Money, Credit and Banking 23, 169-184.

Evans, M., Wachtel, P., 1993. Inflation regimes and the sources of inflation uncertainty. Journal of Money, Credit and Banking 25, 475-511.

Fiorentini, G., Maravall, A., 1996. Unobserved components in ARCH models: An application to seasonal adjustment. Journal of Forecasting 15, 175-201.

Fiorentini, G., Sentana, E., Shephard, N., 2004. Likelihood estimation of latent generalized ARCH structures. Econometrica, 72, 1481-1517.

Harvey, A.C., 1989. Forecasting, structural Time Series Models and the Kalman Filter. Cambridge University Press, Cambridge.

Harvey, A.C., Koopman, S., 1992. Diagnostic checking of unobserved-components time series models. Journal of Business and Economic Statistics 10, 377-389.

Harvey, A.C., Ruiz, E., Sentana, E., 1992. Unobserved component time series models with ARCH disturbances. Journal of Econometrics 52, 129-157.

Hasbrouck, J., 1999. Security Bid/Ask dynamics with discreteness and clustering. Journal of Financial Markets $2,1-28$.

He, C., Teräsvirta, T., 1999. Properties of moments of a family of GARCH processes. Journal of Econometrics 92, 173-192.

Hentschel, L.F., 1995. All in the family: nesting linear and non linear GARCH models. Journal of Financial Economics 39, 139-164.

Kim, C.J., 1993. Unobserved-component time series models with Markov-switching heteroskedasticity: Changes in regime and the link between inflation rates and inflation uncertainty. Journal of Business and Economic Statistics 11, 341-349.

King, M., Sentana, E., Wadhwani, S., 1994. Volatility and links between national stock markets. Econometrica 62, 901-933.

Koopman, S.J., Harvey, A.C., Doornik, J.A., Shephard, N., 2000. Stamp: Structural time series analyser, modeller and predictor. Timberlake Consultants Press, London.

Koopman, S.J., Bos, C.S., 2004. State space models with common stochastic variance. Journal of Business and Economic Statistics, 22, 346-357.

Maravall, A., 1983. An application of nonlinear time series forecasting. Journal of Business and Economic Statistics $1,66-74$.

Maravall, A., 1987. Minimum mean squared error estimation of the noise in unobserved component models. Journal of Business and Economic Statistics 5, 115-120.

Morgan, Trevor, 1999. Limit moves as censored observations of equilibrium futures price in GARCH processes. Journal of Business and Economic Statistics 17, 397-408.

Nelson, D.B., 1991. Conditional heteroscedasticity in asset returns: a new approach. Econometrica 59, 347-370.

Ord, J.K., Koehler, A.B., Snyder, R.D., 1997. Estimation and prediction for a class of dynamic nonlinear statistical models. Journal of the American Statistical Association 92, 1621-1629.

Pérez, A., Ruiz, E., 2003. Properties of the sample autocorrelations of non-linear transformations in long memory stochastic volatility models. Journal of Financial Econometrics 1, 420-444.

Rodriguez, J., Ruiz, E., 2003. A powerful test for conditional heteroscedasticity for financial time series with highly persistent volatilities. WP03-67(16), Universidad Carlos III de Madrid. 
Sentana, E., 1995. Quadratic ARCH models. Review of Economic Studies 62, 639-661.

Sentana, E., Calzolari, G., Fiorentini, G., 2004. Indirect estimation of conditionally heteroskedastic factor models, Manuscript.

Shephard, N., 1996. Statistical aspects of ARCH and stochastic volatility. In: Barndorff-Nielsen, O.E., Cox, D.R., Hinkley, D.V. (Eds.), Statistical Models in Econometrics, Finance and other Fields. Chapman \& Hall, London, pp. 1-67.

Wei, S.X., 2002. A censored GARCH model asset returns with price limits. Journal of Empirical Finance 9, 197-223. 